\title{
Spatial and Temporal Variability of ECG Waveforms Observed in Sinus Rhythm and During Atrio-Ventricular Stimulation in a Patient with Implanted ICD: Case Study
}

\author{
M Fereniec ${ }^{1}$, U Steinhoff ${ }^{2}$, D Janusek ${ }^{3}$, A Przybylski ${ }^{4}$, R Maniewski $^{1}$ \\ ${ }^{1}$ Institute of Biocybernetics and Biomedical Engineering PAS, Warsaw, Poland \\ ${ }^{2}$ Physikalisch-Technische Bundesanstalt, Berlin, Germany \\ ${ }^{3}$ Institute of Radioelectronics, Warsaw University of Technology, Poland \\ ${ }^{4}$ II Clinic of Coronary Arterial Disease, National Institute of Cardiology, Warsaw, Poland
}

\begin{abstract}
Measurements of surface HR ECG were carried out in an unshielded environment. Signals were acquired from one patient with implanted ICD during sinus rhythm and during atrial and ventricular stimulation. QRS, STT, QRST deflection areas and $T$ wave alternans were studied. Significant differences between two ventricular activation sequences were found in spatial distributions of QRS and STT integrals in comparison to QRST integral maps, of which spatial distribution was almost independent of activation order. Quantification of differences was done with the use of principal component analysis and correlation coefficient between deflection areas of waveforms. Departure maps were also studied. Beat to Beat variations of QRS, STT and QRST deflection areas were calculated and compared to microvolt $T$-wave alternans test results.
\end{abstract}

\section{Introduction}

The constant quest for noninvasive indices which allow for risk assessment of ventricular arrhythmia is justified by a large number of sudden cardiac death (SCD) accidents, where ventricular arrhythmias are one of the main reasons of SCD [1]. The efforts of many medical scientists and doctors are concentrated on the prediction and the prevention of SCD by different diagnostic tools and therapies. The ICD (implantable cardioverter-defibrillator) therapy is of great importance in prevention of SCD nowadays. However the qualification to ICD therapy is still not an easy task. Noninvasive parameters quantifying depolarization and repolarization process, both in time and space, are still searched in spite of existing abundance of their kinds.

This case study allows for noninvasive analysis of variability of ECG waveforms, both in time and in space, since body surface potential mapping (BSPM) was performed together with the recording of orthogonal XYZ leads used for microvolt $\mathrm{T}$ wave alternans (MTWA) calculation. The quantitative assessment of variability of the QRS, STT and QRST integrals with altered activation sequence in a patient with implanted ICD was of special interest in that study. The relationship, between the microvolt $\mathrm{T}$-wave alternans ratio, which reflects temporal heterogeneity of repolarization phase and the parameters assessing spatial heterogeneity of repolarization phase, was also considered.

\section{Methods}

A patient with an old inferior myocardial infarction (MI), with coronary artery disease (CAD), ejection fraction (LVEF) lower than 35\% and with implanted ICD was examined. The ICD was implanted because of nonsustained episodes of ventricular tachycardia (VT). Recordings were made at the Warsaw Institute of Cardiology.

The system used for BSPM consists of 32 active electrodes (BioSemi). By integrating the first amplifier stage with a sintered $\mathrm{Ag}-\mathrm{AgCl}$ electrode, low-noise measurements free of interference are possible. The active electrode is a sensor with very low output impedance, problems with regards to capacitive coupling between the cable and sources of interference, as well as any artefacts by cable and connector movements are significantly reduced [2]. Data were acquired with $16 \mathrm{kHz}$ sampling frequency, and saved to disk after decimation filtering which reduce sampling frequency to $4 \mathrm{kHz}$. BSPM recordings were made in the unshielded environment. Leads were placed on thorax surface according to Lux optimal lead set [3].

The ECG was recorded simultaneously from bipolar, orthogonal XYZ leads, for two minutes, during ventricular pacing at 100 beats per minute using a high- 
resolution ECG device, Institute of Radioelectronics, Warsaw University of Technology). The ECG signal was amplified (gain 1000), filtered (bandwidth: $0.05 \mathrm{~Hz}$ to $560 \mathrm{~Hz})$ and digitized $(2 \mathrm{kHz}$ sampling frequency, 22 bits resolution). In order to obtain a high-quality signal, lowresistant $\mathrm{Ag}-\mathrm{AgCl}$ electrodes were used and the skin was cleaned with alcohol. The ECG was acquired in a quiet place, at room temperature.

\section{Signal analysis}

In BSPM data baseline drift was eliminated with the use of the high-pass filter $(\mathrm{f}=0.33 \mathrm{~Hz})$ and the sampling frequency was decreased to $1 \mathrm{kHz}$ (decimation filter). To obtain averaged ECG signals the cross-correlation function was calculated between manually chosen pattern of QRS complex and the whole signal. For alignment and averaging beats, which correlation coefficient was higher than 0.98 , were chosen. Additionally, beats with higher values of noise were eliminated. Spatial and temporal heterogeneity of repolarization phase was assessed for two activation sequence: sinus rhythm (43 bpm) and atrio-ventricular stimulation $(60 \mathrm{bpm})$. MTWA were calculated during ventricular pacing at $100 \mathrm{bpm}$.

Averaged signals for all leads were used to assess spatial heterogeneity of repolarization phase. For each lead, the QRS, STT, and QRST integral maps were calculated. The beginning of QRS complex was set as the earliest activation point in all 32 leads and the end of QRS complex was established as the latest one. The T wave beginning was set in relation to the latest QRS end plus $80 \mathrm{~ms}$ and the $\mathrm{T}$ wave end as the latest of all 32 leads.

The QRS, STT and QRST integrals were calculated for each lead as the algebraic sum of ECG values from appropriate wave onset to its offset.

A departure map of the QRST integral for sinus rhythm was calculated in comparison to the mean QRST integral of the healthy subjects group, the sign was inversed [4].

To remove the influence of heart rate on the integrals variability caused by change of activation sequence, the correction of integrals values was done using QT interval value.

Two parameters quantifying variability of QRS, STT and QRST integrals were calculated. The first one, a variability index, was calculated using Principal Component Analysis as described in [5]. The second one was calculated as the correlation coefficient between the same integrals for different activation sequences.

The spatial heterogeneity of microvolt $\mathrm{T}$ wave alternans ratio (MTWA) was assessed from unipolar leads according to formula defined for XYZ leads.

Before calculating microvolt $\mathrm{T}$-wave alternans baseline noise was estimated and removed from the electrocardiogram with the use of cubic spline method [6]. The QRS complexes were identified using an algorithm developed by Pan and Tompkins [7]. T waves locations were estimated using Bazett's formula [8]. In further analysis, ECG signal, consisting of 128 T-waves, each 310 ms long, was used. All disrupted PQRST cycles were excluded from analysis (always an even number of beats was removed). MTWA was analysed in the frequency-domain using the fast Fourier transform (FFT) [9]. Power spectral densities (PSD) were calculated for consecutive samples of T-waves. Basing on the level of an averaged PSD at a frequency of 0.5 cycle/beat an alternans ratio was calculated for $\mathrm{X}, \mathrm{Y}$, and $\mathrm{Z}$ leads.

\section{Results}

The QRS width and QT interval length dependence of heart rate is given in Table 1.

Table 1. The QRS width and QT interval length for two different heart rates

\begin{tabular}{|l|l|l|}
\hline Heart rate & QRS width [ms] & QT int [ms] \\
\hline 43 & 172 & 576 \\
\hline 60 & 202 & 562 \\
\hline
\end{tabular}

The variability of the QRS and especially of the STT integral, between sinus rhythm and atrio-ventricular stimulation, was higher than variability of the QRST integral in examined ICD patient, as it was expected from the previous experiments on dogs [5].

The values of variability index of separate integrals are shown in Table 2.

Table 2. The variability index of integrals between activation sequence

\begin{tabular}{|l|l|l|}
\hline \multicolumn{3}{|l|}{ Variability Index $\left[\mathrm{mV}^{2} \mathrm{~ms}^{2} \times 10^{4}\right]$} \\
\hline QRS & STT & QRST \\
\hline 95.31 & 101.97 & 3.37 \\
\hline
\end{tabular}

The kind of variability between integrals calculated for different activation sequence is expressed by correlation coefficient shown in Table 3.

Table 3. Correlation coefficient of separate integrals between sinus rhythm and atrio-ventricular stimulation

\begin{tabular}{|l|l|}
\hline \multicolumn{2}{|l|}{ Correlation_coefficient : } \\
\hline QRS & 0.63 \\
\hline STT & -0.81 \\
\hline QRST & 0.97 \\
\hline
\end{tabular}

The correlation coefficient value for the QRS integral shows that there is low similarity between QRS integral distributions of altered activation sequences and high, 
inverse relation between the STT integral distributions. For the QRST integral the correlation coefficient is very high, what shows that the QRST integral distributions for both activation sequences are almost identical.

The integral maps are shown in Figures 1-3.

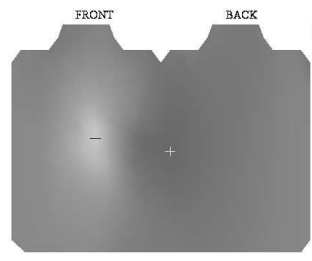

a.)

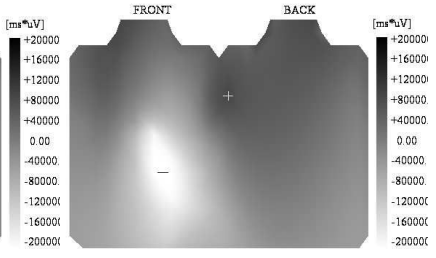

b.)
Figure 1. The QRS integral: a.) sinus rhythm, b.) atrioventricular activation

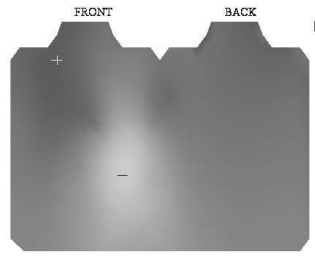

a.)

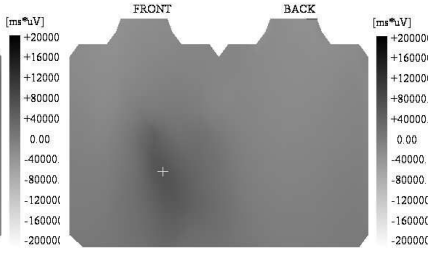

b.)
Figure 2. The STT integral: a.) sinus rhythm, b.) atrioventricular activation

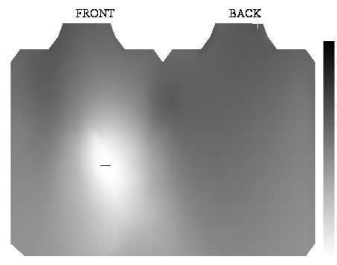

a.)

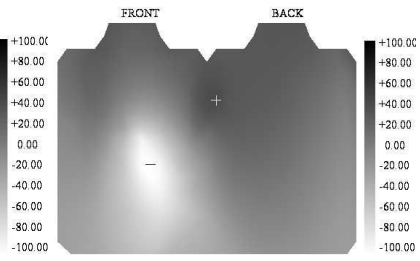

b.)
Figure 3. The QRST integral: a.) sinus rhythm, b.) atrioventricular activation

The alternans ratio, calculated for bipolar XYZ leads equals: in lead $\mathrm{X}-110$, in lead $\mathrm{Y}-40$ and in lead $\mathrm{Z}$ 233. The calculated values significantly exceed threshold which value is 2.5 , established in literature as the critical value separating patients prone to ventricular arrhythmia from patients without threaten of ventricular arrhythmia [6].

The spatial distribution of MTWA ratio calculated for each unipolar lead is presented in Fig. 4.

The values of TWA ratio are clearly higher than prognostic threshold established for XYZ leads. The highest values are observed in the precordial area.

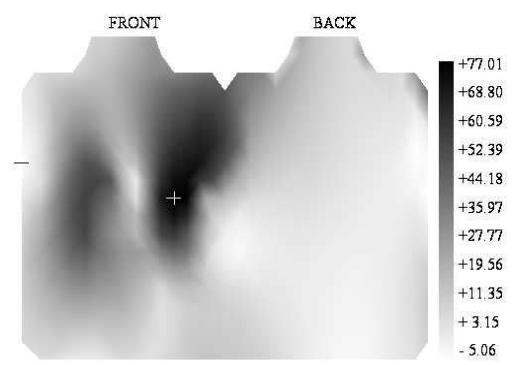

Figure 4. The $\mathrm{T}$ wave alternans ratio distribution calculated for ventricular stimulation at $100 \mathrm{bpm}$.

In the same precordial area the highest values are observed in departure map of QRST integral (Figure 5).

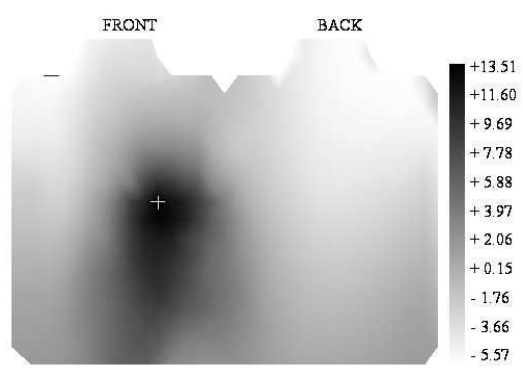

Figure 5. QRST integral departure map calculated for sinus rhythm. 2.

The normal range of departure index varies from -2 to

\section{Discussion and conclusion}

The activation sequence does influence both the QRS integral and the STT integral what is observed in high values of variability index, the nature of that variability is different in both cases. The QRS integral spatial distribution alters with activation sequence, as shown by not high, positive value of correlation coefficient. Th STT integral spatial distribution alters too, but the correlation coefficient, calculted between the STT integrals of two activation sequences, has a high, negative value, what can indicate similarity between spatial distributions of STT integral values for both sequences but inverse polarization.

The variability of QRST integral is clearly smaller than in case of the QRS or the STT integrals, what is explained by Wilson ventricular gradient theory and cancellation of the effects of activation sequence. The ventricular gradient would be zero if the action potentials were identical throughout the myocardium [10].

Nonzero ventricular gradient implies a spatial variation in the form of the action potential, especially of non-uniform recovery properties [10] as the QRST integral is considered to be determined by intrinsic 
ventricular recovery properties [5].

The MTWA ratio was very high, both in XYZ leads and in unipolar leads. The spatial distribution of TWA ratio values were high in the precordial area, where high values were observed in departure map of the QRST integral calculated during sinus rhythm. However it is hard to draw any conclusion on that similarity - if it is accidental or if there is some information behind it.

This case study is an attempt to assess the repolarization dispersion both in spatial and temporal space. To explain, confirm or reject obtained, interesting results further research with larger number of patients is needed.

\section{Acknowledgements}

We would like to thank to Mrs. Anna Zbieć for her invaluable help in the measurements.

\section{References}

[1] Priori SG, Aliot E, Blomstrom-Lundqvist C, Bossaert L, Breithardt G, Brugada P, et al. Update of the guidelines on sudden cardiac death of the European Society of Cardiology. Eur Heart J 2003;24:13-15.

[2] MeetingVanRijn AC, Kuiper AP, Dankers TE, Grimbergen CA. Low-cost active electrode improves the resolution in biopotential recordings. Proc. of the 18th Annual International Conference of the IEEE Engineering in Medicine and Biology Society. Amsterdam 1996, Track1.2.3-3.
[3] Lux RL, Burgess MJ, Wyatt RF, Evans AK, Vincent GM, Abildskov JA. Clinically practical lead systems for improved electrocardiography: comparison with precordial grids and conventional lead systems. Circulation 1979;59:356-63.

[4] Fereniec M, Kępski R, Karpiński G, Walczak F., Opolski G, Maniewski R. Assessment of spatial disparity of repolarization phase by HRECG maps in patients qualified to ICD therapy. Folia Cardiol 2005;12 supp D.

[5] Lux RL, Urie PM, Burgess MJ, Abildskov JA. Variability of the body surface distributions of QRST, ST-T and QRST deflection areas with varied activation sequence in dogs. Cardiovasc Res, 1980;14:607-12.

[6] Mayer CR, Keiser HN. Electrocardiogram baseline noise estimation and removal using cubic splines and state-space computation techniques. Comput Biomed Res 1977; 10:459-70

[7] Pan J, Tompkins WJ. A real-time QRS detection algorithm. IEEE Trans Biomed Eng 1985;32:158-67.

[8] Bazett H. An analysis of the time-relation of electrocardiogram. Heart 1920;7:353-70.

[9] Rosenbaum D, Jackson L, Smith J, et al. Electrical alternans and vulnerability to ventricular arrhythmias. N Engl J Med 1994;330:235-41.

[10] Geselowitz DB. The ventricular gradient revised: relation to the area under the action potential. IEEE Trans Biomed Eng 1983;BME-30:76-8.

Address for correspondence

Małgorzata Fereniec Institute of Biocybernetics and Biomedical Engineering PAS ul. Trojdena 4, 02-109 Warsaw, Poland malgosia@ibib.waw.pl 